\title{
Distribution of erosions in hands and feet at the time for the diagnosis of RA and during 8-year follow-up
}

\author{
Maria L. Andersson ${ }^{1,2}$ (D) B. Svensson ${ }^{1}$ (D) $\cdot$ K. Forslind ${ }^{1,2}$ (I) \\ Received: 14 September 2020 / Revised: 5 October 2020 / Accepted: 12 October 2020 / Published online: 23 October 2020 \\ (C) The Author(s) 2020
}

\begin{abstract}
Background Joint destruction in rheumatoid arthritis (RA) is usually evaluated by radiographs of both hands and feet, while the inflammatory status mostly is evaluated by DAS28 which, however, does not include the feet.

Objectives To investigate the distribution of erosions in hands and feet in early RA over 8 years and its potential clinical implications. Furthermore, the group of patients never showing erosions has been addressed.

Methods This study comprises 1041 patients from the BARFOT study of patients with early RA. Radiographs of hands and feet were performed at baseline, 1, 2, 5, and 8 years and evaluated by the Sharp van der Heijde scoring (SHS) method (32 joints in the hands and 12 in the feet). Disease activity was measured by DAS28, SR, CRP, and function with HAQ.

Results In the feet, there were significantly more eroded joints in percent of examined joints than in the hands at all time points. Patients with erosions only in the feet were younger, more often seropositive and smokers. They had significantly lower baseline DAS28, than the patients with erosions only in the hands. The patients without erosions over time were, at diagnosis, significantly younger and less frequently seropositive compared with patients having erosions.

Conclusions This study highlights the importance of evaluating the feet in patients with RA, both with clinical examinations and with imaging and lends support to the notion that seropositivity and smoking are risk factors for erosive disease. Further studies of patients with nonerosive disease are needed.

\section{Key Points:}

- Foot problems are common in RA

- This study emphasizes the limitations of DAS28 and Sharp van der Heijde score as regards evaluating disease activity and radiographic damage

- This study highlights the importance of evaluating the feet in patients with RA with clinical examinations and imaging

- This study also points out the need of further studies of patients with non-erosive RA.
\end{abstract}

Keywords Disease activity $\cdot$ Distribution of erosions $\cdot$ No erosions $\cdot$ Radiography $\cdot$ Rheumatoid arthritis

Publish as a poster at EULAR 2017 (Annals of the Rheumatic Diseases, volume 76, supplement 2, year 2017, p. 1136)

Supplementary Information The online version contains supplementary material available at https://doi.org/10.1007/s10067-02005465-x.

Maria L. Andersson

maria.andersson@fou-spenshult.se

1 Faculty of Medicine, Department of Clinical Sciences, Rheumatology, Lund University, Lund, Sweden

2 Spenshult Research and Development Centre, Halmstad, Sweden

\section{Introduction}

Rheumatoid arthritis (RA) is a systemic, chronic, and progressive inflammatory disease, characterized by joint swelling, tenderness, and destruction of synovial joints and eventually irreversible loss of physical function [1]. It has since long been recognized that presence of bone erosions is a hallmark of RA, driven by the inflammatory process resulting in various degrees of joint destruction and disability.

The distribution of erosions at the diagnosis of RA may vary and are most commonly detected in the hands and/or feet. Accordingly, the methods for scoring erosions are based on conventional radiographs of hands and feet. 
Involvement of the feet is very common in early RA patients [2], with the forefoot (metatarsophalangeal joints) usually being the first anatomical location where the symptoms are noticed [3-5]. At diagnosis, up to 50\% of patients present some kind of foot problems [6] and continuing foot involvement in patients with longstanding RA has been estimated to $30-90 \%[2,7,8]$.

In a number of patients, no erosions are detected at diagnosis. However, in a few cases, erosions are never detected, not even after several years. Whether this is consistent with the concept of RA does not seem to be fully established.

The main goal of the management of RA is to suppress the disease activity in order to reach a state of remission [9]. However, clinical remission criteria may overlook important aspects of RA, especially subclinical inflammatory activity and continued progression of radiographic joint damage [10, 11].

Since long, the 28 joint Disease Activity Score (DAS28), a well validated composite index, is widely used to assess disease activity in RA [12,13]. However, a potential drawback is that this measure does not include the feet.

To our knowledge, the distribution of erosions and its possible clinical implications have previously not been addressed. Therefore, we have performed a study on 1041 patients from the BARFOT early RA cohort to explore this issue. The aims were to investigate the distribution of erosions in hands and feet in early RA over 8 years, to elucidate if the distribution might have clinical implications, e.g., on the interpretation of DAS28 in patients with a predominance of feet erosions. Furthermore, the group of patients never showing erosions, neither at diagnosis nor at follow-up, has been addressed.

\section{Material and methods}

\section{Patients}

In all, 2857 patients were included in the BARFOT (Better Anti-Rheumatic FarmacOTherapy) study from 1992 to 2006. Patients were $\geq 18$ years of age, fulfilling the classification criteria for RA established by the American Rheumatism Association [14] and had a disease duration of $\leq 12$ months. The 1041 patients who had radiographs at diagnosis with available separate data of hands and feet and at least two of four follow-ups during 8 years were included in this study. At 8 years, 842 patients participated in the follow-up; 705 of these had available radiographs.

The patients were assessed according to a structured protocol at baseline, 3 and 6 months, and at 1,2, 5, and 8 years. The patients were treated with DMARDs in accordance with the recommended treatment strategy in Sweden as earlier described $[15,16]$.

\section{Clinical disease assessments}

Disease activity was assessed by the composite index Disease Activity Score calculated on 28 joints (DAS28; range 0-9.4, best to worse) [12], C-reactive protein (CRP), and the erythrocyte sedimentation rate (ESR; $0-150 \mathrm{~mm} / \mathrm{h}$ ), analyzed by the Westergren method [17]. DAS28 includes the number of swollen joints (range 0-28), number of tender joints (range 0 28), and patient's global assessment of disease activity (PatGA) measured on a visual analogue scale (VAS). Pain was assessed by a VAS (ranged 0-100 $\mathrm{mm}$, best to worse).

Physician's global assessment of disease activity (PhAss) was assessed by a 5 graded Likert scale, ranging from no disease activity to high disease activity.

Rheumatoid factor (RF) was measured according to the current laboratory standards at the participating hospitals. Antibodies to cyclic citrullinated peptides (antiCCP) were detected using the ELISA CCP2 test (Euro-Diagnostica, Malmö, Sweden).

The Swedish version of the Stanford Health Assessment Questionnaire (HAQ) was used to measure daily life function (range 0-3, best to worse) [18].

\section{Radiographic assessments}

Posterior-anterior radiographs of the hands and feet were assessed at baseline and at 1, 2, 5, and 8 years according to the van der Heijde modification of the Sharp score (SHS) where 32 joints in the hands and 12 in the feet are assessed [19], calculating total SHS (range 0-448), erosion score (ES) (range 0-280), and joint space narrowing score (JSN) (range 0-168). Erosive disease was defined as presence of erosions on radiographs of the hands (hands and wrists) and feet at baseline, erosion score $\geq 1$. Eroded joints were also assessed in percent of examined joints and percent of maximum erosion score. The films were read by one of two experienced readers. Double readings of a fraction of films showed good agreement between the two readers. The intraclass correlation coefficient for SHS was excellent $(0.940-0.998)$.

\section{Statistics}

Statistical analyses were performed using SPSS version 21.0 statistical software (IBM SPSS). To test the differences between groups, the independent samples $T$ test was used for continuous variables, and the chi-square test was used for proportions. When comparing more than two groups, ANOVA post hoc (Tukey) analyses were performed. All significance tests were 2-tailed and conducted at the 0.05 level of significance. 


\section{Results}

\section{Demographic and clinical characteristics}

Demographic and clinical characteristics for all 1041 patients at diagnosis are shown in Table 1 . The mean age was 56 years and $68 \%$ were women, $68 \%$ were seropositive (RF and/or antiCCP positive), and $27 \%$ were current smokers. The mean disease duration was 6 months, DAS28 5.06, pain VAS 45, ESR 32, HAQ 0.94, and erosion score 2.

After 8 years, 199 patients were lost to follow-up, of whom $58 \%$ were women. These patients were at diagnosis older, had higher mean ESR and HAQ as well as radiographic scores.

The clinical characteristics of the 842 patients, who completed the 8-year follow-up, are shown in Table 1. The mean DAS28 was 2.93, pain VAS 30, ESR 17, HAQ 0.61, and erosion score 8.

\section{Distribution of erosions in hands and/or feet}

The patients were divided into four groups according to the distribution of erosions at diagnosis - no erosions (57\%), erosions only in the hands (18\%), erosions only in the feet (10\%), and erosions in both hands and feet (15\%).

At diagnosis, significant differences were found between the groups regarding age, seropositivity, current smoking, DAS28, PhAss, ESR, pain VAS, SHS, ES and JSN but not regarding gender, disease duration, PatGA, CRP, or HAQ (Table 1).

The mean SHS was consistently higher in the hands (Fig. 1a) than in the feet but when the mean number of eroded joints was calculated in percent of examined joints, the feet had significantly more erosions than the hands at all time points during the observation years (Fig. 1b), and the feet also had higher erosion score in percent of maximum erosion score at 8 years (data not shown). Figure 1c and d shows the distribution of erosions in percent of examined joints over 8 years in hands and feet in the different groups.

Comparisons between the groups at diagnosis revealed significant differences between the groups regarding some demographic and clinical characteristics (Table 2). These were of particular interest in the groups "feet only" and "no erosions" and will be described separately.

There were no significant differences between the groups as to the presence of reported arthritic symptoms from large joints at diagnosis (data not shown).

The groups did not differ significantly as to treatment given at diagnosis, not regarding cDMARDs, bDMARDs, or corticosteroids $(p=0.37)$.

The distribution of erosions after 8 years is shown in Fig. 2. Of the patients with no erosions at diagnosis, $47 \%$ still had no erosions after 8 years. More than half of the patients with erosions in hands or feet only had erosions in both hands and feet while about onethird of them did not change location after 8 years.
The clinical (845 patients) and radiological (705 patients) outcomes at the 8-year follow-up visit in the four erosions distribution groups are shown in Table 1 with significant differences between the groups only regarding ESR, CRP, ES, JSN, and SHS.

A post hoc analysis (Table 2) revealed that the group with erosions in both hands and feet had significantly higher ESR than the no erosions and feet only groups and had also significantly higher CRP than the no erosion group.

The no erosions group had significantly lower radiographic scores than the other groups. There were no significant differences between the groups regarding DAS28, PatGA, pain, or HAQ.

Furthermore, no significant differences were noted between the groups regarding frequency of remission or persistent disease activity (data not shown).

There were no group differences over the 8 years in treatment with cDMARDs, bDMARDs, or corticosteroids (CS) (data not shown).

Repair of erosions is likely to have occurred in patients with erosions at diagnosis, who showed no erosions after 8 years, and in some cases, an erosion might have disappeared while another was new, in another location. This occurred in at least 47 patients (Fig. 2)

\section{Patients with erosions only in the feet}

The mean erosion score at diagnosis was 3.1 in the group with erosions in feet only vs 3.4 in the group with hands only, nonsignificant. However, there were significantly more eroded joints in the feet than in the hands, in percent of examined joints, mean (SD) 4.7 (10.9) vs. 3.2 (6.1) (Fig. 1b).

During the disease course up to 8 years, the mean number of eroded joints in percent of examined joints was consistently and significantly higher in the feet only group than in the hands only group (Fig. 1c and d).

At diagnosis, the patients with erosions only in the feet were, compared with those with erosions only in the hands, significantly younger ( 52 vs 62 years), were more frequently smokers (47 vs $28 \%$ ), and more often seropositive (85 vs $69 \%$ ).

The feet only group had the lowest value for DAS28. The mean DAS28 differed significantly between the groups with different locations of the erosions at diagnosis $(p=0.024)$. In the post hoc tests, the feet-only group differed significantly from the group with no erosions $(p=0.048)$ and that with erosions only in the hands ( $p=0.015)$ (Tables 1 and 2). There was no statistically significant difference between the group with erosions only in the feet and the group with erosions in both hands and feet $(p=0.074)$. Except for these significant differences in DAS28 between groups at diagnosis, no significant differences in DAS2 8 were seen during the disease course or after 8 years. 


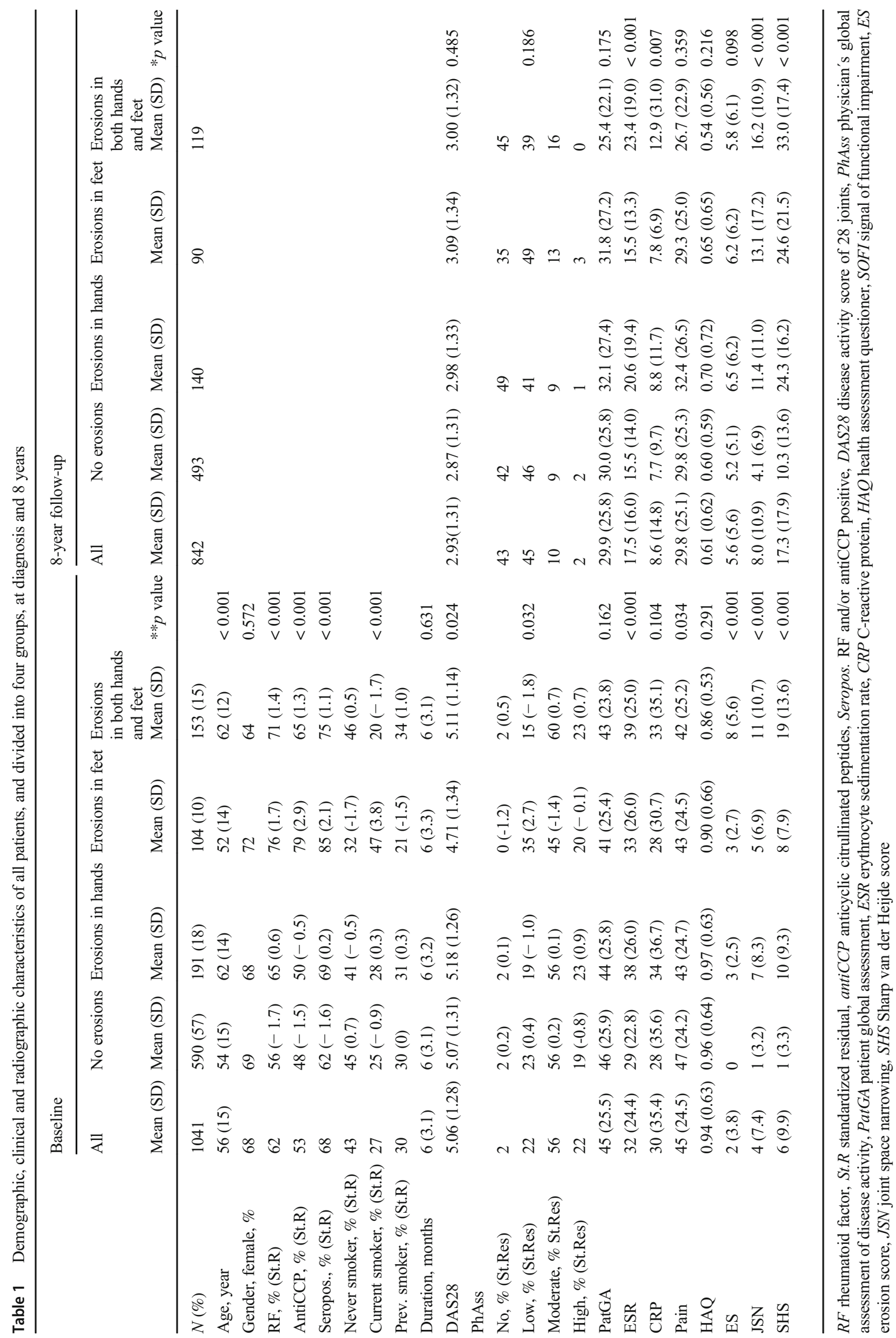


a

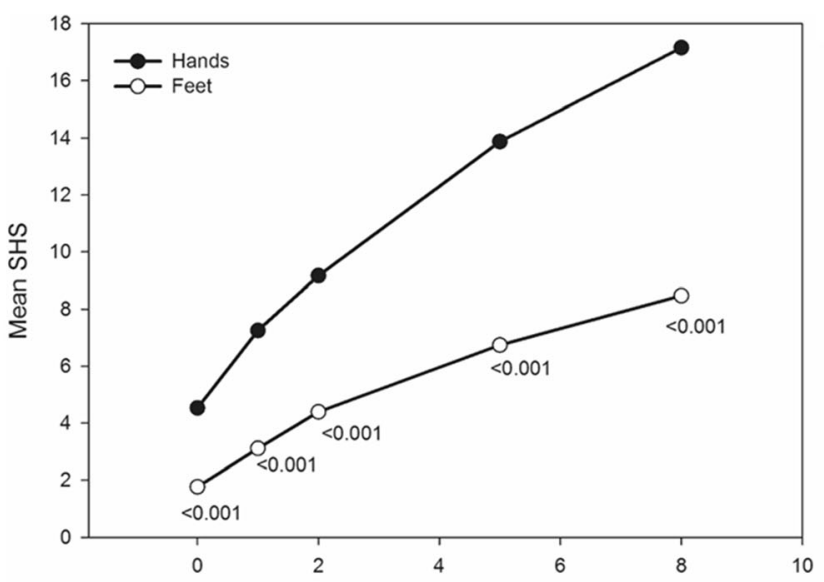

C $\frac{0}{0}$

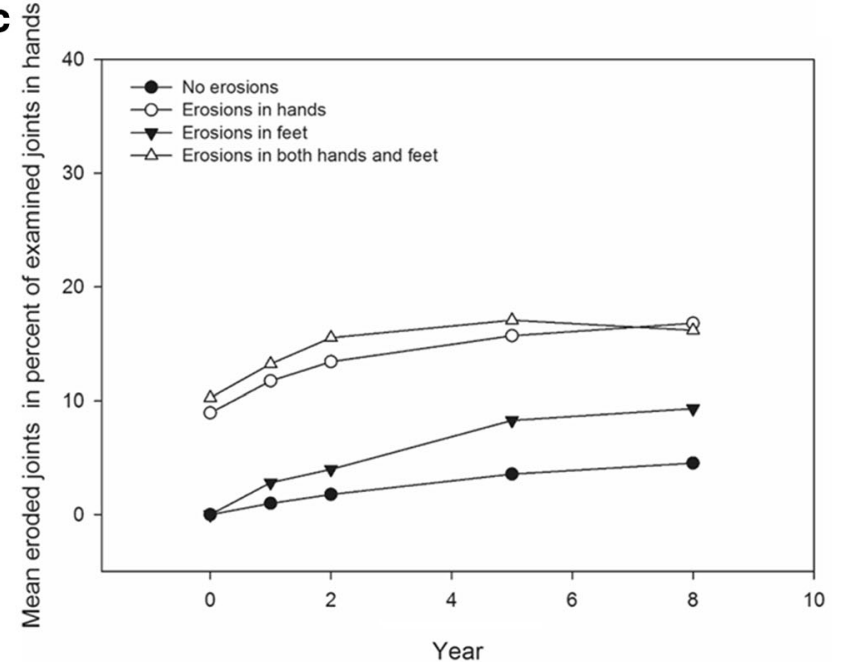

b

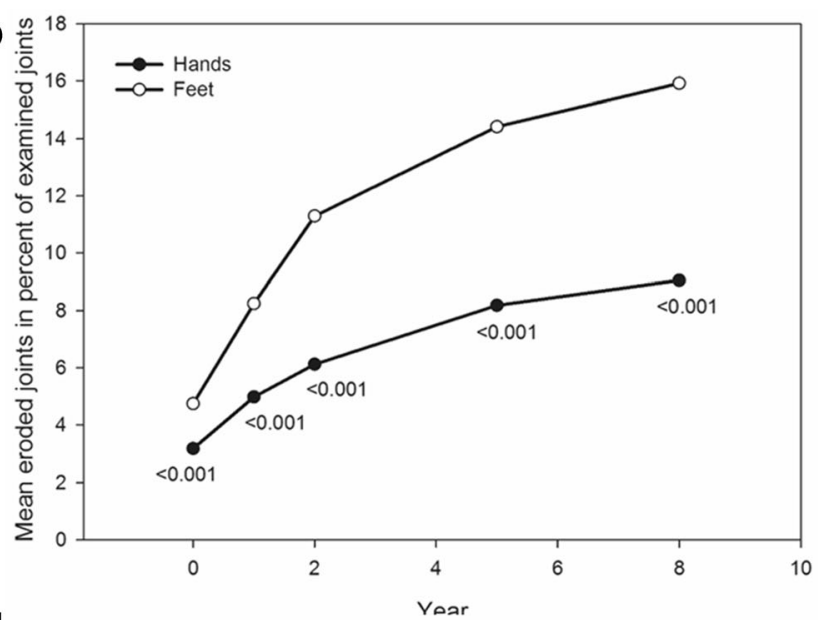

$\mathbf{d}_{\stackrel{\mathbb{\Phi}}{\Phi}}$

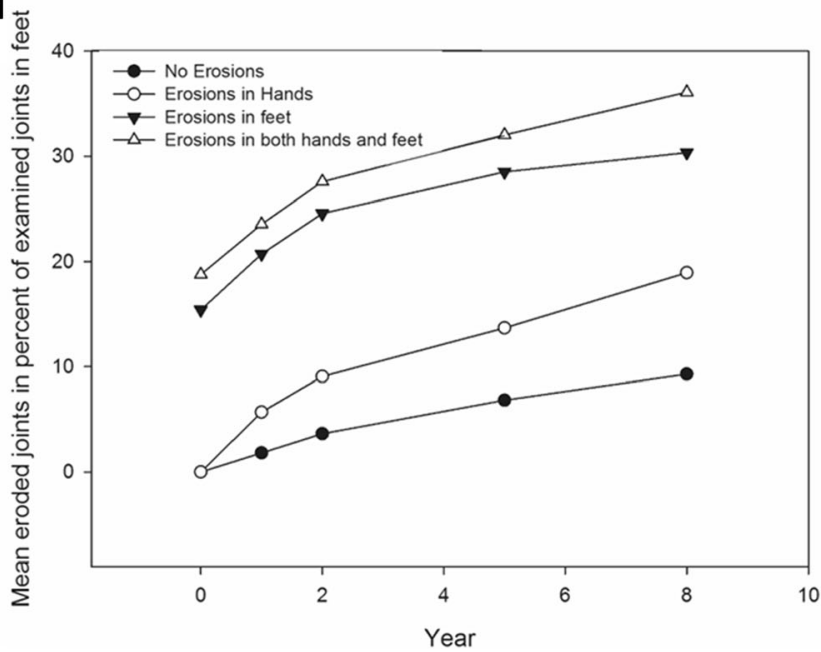

Fig. 1 Mean Sharp van der Heijde score (SHS) (a), eroded joints in percent of examined joints in hands and feet during 8 years (b) and eroded joints in percent of examined joints in hands (c) and feet (d) in the groups divided by erosions at diagnosis during 8 years

After 8 years, the groups differed in levels of ESR, CRP, and radiographic scores (Table 1). The post hoc analysis revealed that the feet only group had a lower mean ESR and CRP similarly to that in the no erosions group (Table 2). The hands and feet erosion group had significantly more erosions than the feet only and hands only groups.

The treatment with DMARDs or CS in the feet only group did not differ from that in the other groups, neither at diagnosis nor during the disease course or after 8 years (data not shown).

\section{Patients who never had any erosions compared with those who had erosions occasionally or consistently}

Of the 1041 patients, 545 had radiographs performed on all five occasions and were studied to compare the patients who never had erosions with those who had erosions occasionally or consistently during the 8-year follow-up. These 545 patients were significantly younger, had a lower ESR and HAQ, fewer erosions and lower SHS at diagnosis compared with the patients lacking radiographs at one or more of the predetermined follow-up visits (data not shown).

The 545 patients were divided into two groups, the never erosive group ( $n=138)$ and the group, who showed erosions on some or all assessments, the ever erosive group $(n=407)$.

At diagnosis, some significant differences between the groups were noted. Thus, compared with the ever erosive group, the patients in the never erosive group were significantly younger, were less frequently seropositive, and had more tender joints but lower ESR and CRP (Table 3).

After 8 years, the clinical differences between the never and ever erosive groups were similar to those at diagnosis. Thus, ESR was still significantly lower, the tender joint count higher and now the swollen joint count was lower in the never erosive group (Supplement figure 1). At diagnosis, the radiological scores were significantly lower in the never erosive group (Table 3).

No significant differences were noted between the never and ever erosive groups regarding frequency of remission or persistent disease activity (data not shown). 
Table 2 Post hoc comparisons between the groups of patients with different distribution of erosions at diagnosis and at 8 years

\begin{tabular}{lllllll}
\hline & $\begin{array}{l}\text { Group 1 } \\
\text { No erosions } \\
\text { mean (SD) }\end{array}$ & $\begin{array}{l}\text { Group 2 } \\
\text { Hands only } \\
\text { mean (SD) }\end{array}$ & $\begin{array}{l}\text { Group 3 } \\
\text { Feet only } \\
\text { mean (SD) }\end{array}$ & $\begin{array}{l}\text { Group 4 } \\
\text { Hands and } \\
\text { feet mean (SD) }\end{array}$ & $\begin{array}{l}\text { ANOVA } \\
p \text { value }\end{array}$ & $\begin{array}{l}\text { Tukey post hoc analysis } \\
\text { Groups significantly different }\end{array}$ \\
\hline Age, years & $54(15)$ & $62(14)$ & $52(14)$ & $62(12)$ & $<0.001$ & $1+2 ; 1+4 ; 2+3 ; 3+4$ \\
DAS28 (0) & $5.07(1.31)$ & $5.18(1.26)$ & $4.71(1.34)$ & $5.11(1.14)$ & 0.024 & $1+3,2+3$ \\
ESR(0) & $29(22.8)$ & $38(26.0)$ & $33(26.0)$ & $39(25.0)$ & $<0.001$ & $1+2 ; 1+4 ;$ \\
Pain (0) & $48(24.2)$ & $44(24.7)$ & $44(24.5)$ & $42(25.2)$ & 0.034 & $1+2 ; 1+3 ; 1+4 ; 2+4 ; 3+4$ \\
ES (0) & 0 & $3(2.5)$ & $3(2.7)$ & $8(5.6)$ & $<0.001$ & $1+2 ; 1+3 ; 1+4 ; 2+3 ; 2+4 ; 3+4$ \\
JSN (0) & $1(3.2)$ & $7(8.3)$ & $5(6.9)$ & $11(10.7)$ & $<0.001$ & $1+2 ; 1+3 ; 1+4 ; 2+3 ; 2+4 ; 3+4$ \\
SHS (0) & $1(3.3)$ & $10(9.3)$ & $8(7.9)$ & $19(13.6)$ & $<0.001$ & $1+2,1+4,3+4$ \\
ESR 8 years & $15(14.0)$ & $21(19.4)$ & $15(13.3)$ & $23(19.0)$ & $<0.001$ & $1+4$ \\
CRP 8 years & $8(9.7)$ & $9(11.7)$ & $8(6.9)$ & $13(31.0)$ & 0.007 & $<+2,1+3,1+4,2+4,3+4$ \\
ES 8 years & $4(7)$ & $11(11)$ & $13(17)$ & $16(11)$ & $<0.001$ & $1+2,1+3,1+4,2+4,3+4$ \\
JSN 8 years & $10(14)$ & $24(16)$ & $25(21)$ & $33(17)$ & $<0.001$ & $1+2,1+3,1+4,2+4,3+4$ \\
SHS 8 years & $14(18)$ & $36(24)$ & $38(36)$ & $49(24)$ & $<0.001$ & $1+3$ \\
\hline
\end{tabular}

DAS28 disease activity score of 28 joints, ESR erythrocyte sedimentation rate, ES erosion score, JSN joint space narrowing, SHS Sharp van der Heijde score

There were distinct differences in DMARD treatment between the groups (Table 3 ). Thus, bDMARDs and cDMARDs were invariably less frequently given to patients who never showed erosions. In contrast, prednisolone was used in fairly similar proportions to patients in the two groups.

\section{Patients who never had any erosions compared with those with no erosions at diagnosis but erosions later during the disease course}

Of the 545 patients with radiographs performed on all five occasions, 328 had no erosions at diagnosis, 138 continued without erosions during the disease course (never erosive group) while 190 developed erosions later (later erosive group) during the 8-year period.

At diagnosis, the never erosive group was significantly less frequently seropositive, but otherwise, the two groups were clinically very similar while SHS and JSN were significantly higher in the later erosive group (Table 4).

After 8 years, the groups were clinically very similar although the later erosive group had significantly more swollen joints (Supplement figure 2). This group also showed significantly higher radiographic scores (Table 4).

No significant differences were noted between the never and later erosive groups regarding frequency of remission or persistent disease activity (data not shown).
Fig. 2 The distribution of erosions in hands and feet at diagnosis and at 8 years in the 705 patients with radiographs on both occasions

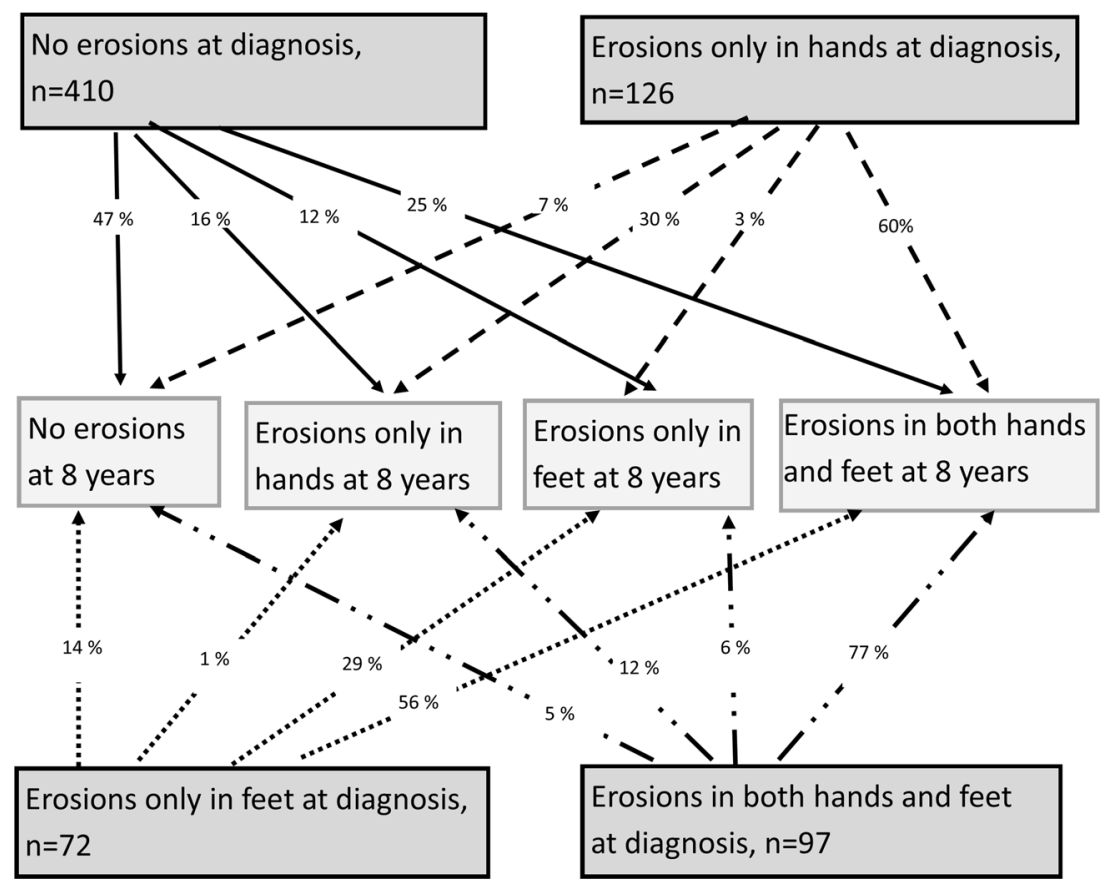


Table 3 Demographic and clinical differences at diagnosis and 8 years between patients without erosions (never erosive) and patients with erosions occasionally or consistently (ever erosive)

\begin{tabular}{|c|c|c|c|c|c|c|}
\hline & \multicolumn{3}{|l|}{ At diagnosis } & \multicolumn{3}{|l|}{ At 8 years } \\
\hline & $\begin{array}{l}\text { Never erosive } \\
\text { mean }(\mathrm{SD})\end{array}$ & $\begin{array}{l}\text { Ever erosive } \\
\text { mean (SD) }\end{array}$ & $p$ value & $\begin{array}{l}\text { Never erosive } \\
\text { mean }(\mathrm{SD})\end{array}$ & $\begin{array}{l}\text { Ever erosive } \\
\text { mean (SD) }\end{array}$ & $p$ value \\
\hline$N(\%)$ & $138(25)$ & $407(75)$ & & $138(25)$ & 407 (75) & \\
\hline Age, year & $52(14.5)$ & $55(13.2)$ & 0.014 & & & \\
\hline Gender, female, $\%$ & 67 & 71 & 0.337 & & & \\
\hline $\mathrm{RF}, \%$ & 44 & 69 & $<0.001$ & & & \\
\hline AntiCCP, $\%$ & 29 & 63 & $<0.001$ & & & \\
\hline Seropos, $\%$ & 53 & 74 & $<0.001$ & & & \\
\hline Never smoker, $\%$ & 45 & 43 & & & & \\
\hline Smoker, \% & 21 & 28 & 0.212 & & & \\
\hline Prev. smoker, \% & 34 & 29 & & & & \\
\hline Duration, months & $6(3.2)$ & $6(3.0)$ & 0.211 & & & \\
\hline DAS28 & $4.92(1.37)$ & $5.01(1.27)$ & 0.468 & $2.86(1.32)$ & $2.91(1.26)$ & 0.739 \\
\hline \multicolumn{7}{|l|}{ PhAss } \\
\hline No, $\%$ & 3 & 1 & & 50 & 42 & \\
\hline Low, $\%$ & 24 & 23 & 0.386 & 43 & 46 & 0.354 \\
\hline Moderate, $\%$ & 58 & 58 & & 7 & 10 & \\
\hline High, $\%$ & 15 & 18 & & 1 & 2 & \\
\hline SJC & $9(5.6)$ & $9(5.8)$ & 0.228 & $1(2.7)$ & $2(3.2)$ & 0.010 \\
\hline TJC & $8(6.9)$ & $7(5.8)$ & 0.031 & $3(4.6)$ & $2(3.4)$ & 0.013 \\
\hline PatGA & $45(26.4)$ & $45(25.7)$ & 0.836 & $30(25.9)$ & $29(25.0)$ & 0.804 \\
\hline ESR & $26(20.8)$ & $32(23.0)$ & 0.004 & $14(15.1)$ & $18(15.1)$ & 0.039 \\
\hline CRP & $23(27.6)$ & $31(35.2)$ & 0.013 & $6(6.4)$ & $9(18.7)$ & 0.102 \\
\hline Pain & $45(25.2)$ & $46(24.6)$ & 0.522 & $30(25.0)$ & $30(24.0)$ & 0.659 \\
\hline HAQ & $0.90(0.66)$ & $0.91(0.59)$ & 0.929 & $0.59(0.55)$ & $0.57(0.59)$ & 0.695 \\
\hline ES & 0 & $2(3.9)$ & $<0.001$ & 0 & $10(10.5)$ & $<0.001$ \\
\hline JSN & $0(1.6)$ & $5(7.9)$ & $<0.001$ & $3(5.2)$ & $22(18.2)$ & $<0.001$ \\
\hline SHS & $0(1.5)$ & $7(10.5)$ & $<0.001$ & $3(5.2)$ & $32(26.1)$ & $<0.001$ \\
\hline \multicolumn{7}{|l|}{ Treatment } \\
\hline No DMARD, \% (Std.Res.) & $26(1.7)$ & $18(-1.0)$ & & $34(2.8)$ & $19(-1.6)$ & \\
\hline cDMARD, \% (Std.Res.) & $57(-1.5)$ & $71(0.9)$ & 0.008 & $53(-1.2)$ & $63(0.7)$ & $<0.001$ \\
\hline bDMARD, \% (Std.Res.) & 0 & 0 & & $5(-2.1)$ & $13(1.3)$ & \\
\hline CS no DMARD, \% (Std.Res.) & $17(1.5)$ & $11(-0.9)$ & & $8(1.3)$ & $5(-0.8)$ & \\
\hline
\end{tabular}

$R F$ rheumatoid factor, AntiCCP anticyclic citrullinated peptides, Seropos. RF and/or antiCCP positive, DAS28 disease activity score of 28 joints, $P h A s s$ physician's global assessment of disease activity, SJC swollen joint count, TJC tender joint count, PatGA patient global assessment, ESR erythrocyte sedimentation rate, $C R P$ C-reactive protein, $H A Q$ health assessment questioner, $E S$ erosion score, JSN joint space narrowing, SHS Sharp van der Heijde score, DMARD disease modifying antirheumatic drugs, $c$ conventional, $b$ biological, $C S$ corticosteroids

There were significant overall differences between the never and later erosive groups regarding DMARD and CS treatment at diagnosis and 8 years (Table 4 ). The treatment showed similar patterns at all follow-ups (data not shown). The post hoc analysis revealed that the patients in the never erosive group were less frequently treated with DMARDs compared with the patients in the later erosive group. The treatment with CS only was not significantly different between the groups.

\section{Discussion}

This longitudinal study aimed to investigate the radiographic distribution of erosions in hands and feet in patients with early RA followed for 8 years. Fifty-seven percent of the patients had no erosions in hands or feet at baseline, and $47 \%$ of these were still erosion-free after 8 years. At baseline $18 \%$ of the patients had erosions in the hands only, $15 \%$ in both hands and 
Table 4 Demographic and clinical differences at diagnosis and 8 years between patients without erosions (never erosive) and patients with no erosions at diagnosis but erosions at 1 year and/or later (later erosive)

\begin{tabular}{|c|c|c|c|c|c|c|}
\hline & \multicolumn{3}{|l|}{ At diagnosis } & \multicolumn{3}{|l|}{ At 8 years } \\
\hline & $\begin{array}{l}\text { Never erosive } \\
\text { mean (SD) }\end{array}$ & $\begin{array}{l}\text { Later erosive } \\
\text { mean (SD) }\end{array}$ & $p$ value & $\begin{array}{l}\text { Never erosive } \\
\text { mean (SD) }\end{array}$ & $\begin{array}{l}\text { Later erosive } \\
\text { mean (SD }\end{array}$ & $p$ value \\
\hline$N(\%)$ & $138(42)$ & $190(58)$ & & $138(42)$ & $190(58)$ & \\
\hline Age, year & $52(14)$ & $52(12)$ & 0.982 & & & \\
\hline Gender, female, $\%$ & 67 & 74 & 0.168 & & & \\
\hline $\mathrm{RF}, \%$ & 44 & 71 & $<0.001$ & & & \\
\hline AntiCCP, \% & 29 & 68 & $<0.001$ & & & \\
\hline Seropos., $\%$ & 53 & 76 & $<0.001$ & & & \\
\hline Never smoker, $\%$ & 45 & 43 & & & & \\
\hline Smoker, $\%$ & 21 & 29 & 0.214 & & & \\
\hline Previous smoker, $\%$ & 34 & 28 & & & & \\
\hline Duration, months & $7(3)$ & $6(3)$ & 0.187 & & & \\
\hline DAS28 & $4.92(1.37)$ & $5.04(1.24)$ & 0.405 & $2.86(1.32)$ & $2.95(1.32)$ & 0.572 \\
\hline \multicolumn{7}{|l|}{ PhAss } \\
\hline No, $\%$ & 3 & 1 & & 50 & 38 & \\
\hline Low, \% & 24 & 23 & 0.543 & 43 & 48 & 0.087 \\
\hline Moderate, $\%$ & 58 & 61 & & 7 & 12 & \\
\hline High, $\%$ & 15 & 14 & & 1 & 3 & \\
\hline SJC & $9(6)$ & $9(6)$ & 0.681 & $1(3)$ & $2(3)$ & 0.005 \\
\hline TJC & $9(7)$ & $8(6)$ & 0.421 & $3(5)$ & $2(4)$ & 0.137 \\
\hline PatGA & $45(26)$ & $47(25)$ & 0.549 & $30(26)$ & $30(25)$ & 0.832 \\
\hline ESR & $26(21)$ & $29(21)$ & 0.223 & $14(15)$ & $16(13)$ & 0.311 \\
\hline CRP & $23(28)$ & $29(25)$ & 0.137 & $6(6)$ & $8(12)$ & 0.066 \\
\hline Pain & $45(25)$ & $50(23)$ & 0.071 & $30(25)$ & $30(24)$ & 0.927 \\
\hline HAQ & $0.90(0.66)$ & $0.98(0.60)$ & 0.304 & $0.59(0.55)$ & $0.55(0.55)$ & 0.472 \\
\hline ES & 0. & 0 & 0.241 & 0 & $7(8)$ & $<0.001$ \\
\hline JSN & $0(2)$ & $1(3)$ & $<0.001$ & $3(5)$ & $15(15)$ & $<0.001$ \\
\hline SHS & $0(2)$ & $1(3)$ & $<0.001$ & $3(5)$ & $22(20)$ & $<0.001$ \\
\hline \multicolumn{7}{|l|}{ Treatment } \\
\hline No DMARD, \% (Std.Res.) & $26(1.1)$ & $18(-1.0)$ & & $34(2.4)$ & $17(-2.1)$ & \\
\hline cDMARD, \% (Std.Res.) & $57(-1.2)$ & $71(1.0)$ & 0.033 & $53(-1.2)$ & $66(1.0)$ & $<0.001$ \\
\hline bDMARD, \% (Std.Res.) & 0 & 0 & & $5(-1.8)$ & $13(1.5)$ & \\
\hline CS no DMARD, \% (Std.Res.) & $17(1.2)$ & $11(-1.0)$ & & $8(1.2)$ & $4(-1.0)$ & \\
\hline
\end{tabular}

$R F$ rheumatoid factor, AntiCCP; anticyclic citrullinated peptides, Seropos. RF and/or antiCCP positive, DAS28 disease activity score of 28 joints, $P h A s s$ physician's global assessment of disease activity, SJC swollen joint count, TJC tender joint count, PatGA patient global assessment, ESR erythrocyte sedimentation rate, $C R P$ C-reactive protein, $H A Q$ health assessment questioner, $E S$ erosion score, JSN joint space narrowing, SHS Sharp van der Heijde score, DMARD disease modifying antirheumatic drugs, $c$ conventional, $b$ biological, $C S$ corticosteroids

feet and $10 \%$ only in the feet. There was no difference in disease duration between the groups.

The Sharp van der Heijde score (SHS) for evaluation of radiographic damage in hands and feet in patients with RA has an overweight for the hands as more joints in the hands than in the feet are included in the score [20]. Thus, SHS does not catch present damage in the feet. However, in this study, we calculated the development of erosions in percent of examined joints and found throughout the study more erosions and progression in the feet than in the hands. This is in agreement with previous studies reporting more erosions in the feet than in the hands, and also that erosions developed earlier in the feet [20-22].

van der Heijde et al. [20] reported in a study of 90 patients that at study start more foot than hand joints were affected, and that this predominance was still present after 3 years. Furthermore, Plant et al. [22] followed 114 patients for 8 years and found that the feet showed the greatest initial radiological 
progression. Hulsman et al. [21] in a study on 502 patients, reported that feet joints, especially the fifth metatarsophalangeal joint, generally became eroded earlier than hand joints.

At diagnosis, $25 \%$ of the patients in this study had erosions in the feet, $10 \%$ of these only in the feet. These patients were younger, more frequently smokers and had more often RF- and/or antiCCP antibodies compared with the other groups. Seropositivity and smoking are well known to be associated with high disease activity, assessed by DAS28 [23]. However, in this study, the group with erosions only in the feet had lower DAS28, despite a high rate of smokers and seropositivity. One explanation to this could be that since DAS28 does not include examination of the feet the disease activity may be underestimated in patients with inflammation mainly localized to the feet.

These observations are in agreement with those in a study by Bakker et al. [24]. They studied 265 RA patients over 5 years and found, in agreement with us, that patients with more radiographic progression in the feet were younger and more often RF-positive [24]. In that study, the patients were divided into different groups according to radiographic progression rate. They found that the patients developing radiographic progression predominantly in the feet showed, in contrast to the patients in the other groups, no corresponding change in DAS28. This was interpreted to be due to an underestimation of the disease activity measured by DAS 28 among foot progressors.

These studies thus suggest that erosions at diagnosis, limited to the feet, may be associated with low values for DAS28 due to the fact that inflammation in the foot joints is not reflected by DAS28. This may be more than a marginal problem since the prevalence of baseline involvement of the feet in early RA is common and may cause undertreatment due to misleadingly low values for DAS28. In addition, patients categorized as being in remission by DAS28 have been found to have inflammation in the feet in up to $40 \%$ [8, 25], further highlighting the importance of also examining the feet. We agree with the statement of Hulsmans et al. [21] and Bakker et al. [24] that radiographs of the feet should be included in assessments of radiologic damage in clinical trials as well as in daily practice.

The patients, who never developed erosions during followup, were younger, less often antiCCP or RF positive and had lower ESR over time than the patients with erosions, always or at times. In addition, the never erosive group received significantly less treatment with DMARDs while corticosteroids were similarly given. Taken together, these observations suggest that the patients who never developed erosions had a milder disease, possibly different from RA. Our results are in agreement with those of Liao et al. [26], who investigated clinical predictors of erosion-free status in rheumatoid arthritis where the patients were stratified by disease duration and followed for 2 years. They found that 56 (21\%) of 271 patients were still erosion-free after 2 years. The mean disease duration at study start was 3.4 years for the erosion-free patients and 4.5 years for the erosive group. The erosion-free patients were younger and less often RF- and/or antiCCP positive.

In the present study, $57 \%$ of the patients had no erosions at diagnosis, and after 8 years, $47 \%$ of these still had no erosions. In other studies, 40 to $50 \%$ of the patients were nonerosive at diagnosis, and about 4-30\% remained nonerosive during follow-up periods for 2 to 10 years [20, 22, 27].

The higher percent of erosion-free patients at follow-up in our study might be due to shorter disease duration at diagnosis and improved treatment as the referred studies all were carried out in the 1980s and early 1990s.

In a cross-sectional study by Amaya-Amaya et al. [28], 110 out of 500 patients with RA were erosive after a follow-up period of median 10 years. Of these, 40 patients with a disease duration of more than 5 years were studied by plain radiographs, ultrasound (US), and computed tomography (CT). Of these only 8 (20\%) were nonerosive by all three methods (by radiography $53 \%$, US $43 \%$, CT $50 \%$ ). Accordingly, they drew the conclusion that nonerosive RA is very rare. They also performed a systematic literature search for studies evaluating nonerosive RA and associated factors. Seventeen studies reporting highly diverging prevalence were retrieved, which may be explained by the great differences between studies in disease duration, ( 3 months to 16 years), followup time (1-12 years) and study design. Factors associated with nonerosions included seronegativity and younger age, which is in agreement with the results in the present study.

Absence of erosions at study end in patients with erosions at diagnosis occurred in 24 patients and may indicate repair. Whether erosion repair occurs or not in RA has earlier been debated, but today this feature has been demonstrated in several studies with different prevalence depending on imaging modality and definition of repair [29].

Foot problems have a negative impact on the quality of life in patients with RA $[25,30]$. As clinically detectable inflammation precedes erosions in the joints [31,32], it is of importance to integrate the feet in the clinical evaluation. The importance of earliest possible antiinflammatory treatment of patients with newly diagnosed RA is well recognized [33, 34], and rapid attainment of remission can prevent or limit joint damage and maintain good quality of life. In order to achieve this, it is important recognize the presence of inflammation also in the feet.

To our knowledge, this is the first study on radiographic damage in RA, in which patients who never had erosions (never erosive) are compared both with patients who occasionally or consistently had erosions (ever erosive) and with those who developed erosions during follow-up (later erosive).

The main differences between the never erosive and the other groups was that the never erosive group was less frequent seropositive and received more seldom treatment with DMARDs. However, the mean DAS28 over time was similar in the groups, which may be explained by the observation that 
the never erosive group still had higher tender joint count compared with the ever and later erosions groups, significant only for the ever erosive group. These observations suggest that patients who never developed erosions may represent a milder disease than those with erosions.

In previous reports, there have been divergent reports regarding associations between radiographic damage and disability measured by HAQ.[35, 36]. In a 5-year study of 191 patients with early RA, Combe et al. [35] found radiological progression in half of the patients whereas HAQ disability improved in most of them, Ödegård et al. [36], on the contrary, found in a 10-year study that radiographic damage contributed to impaired physical function as did Andersson et al. [37] in a study of 1938 patients. Of interest, Maillefert et al. [38] followed 135 patients for 5 years and found changes in joint damage to be related to subsequent HAQdisability due to changes in joint narrowing rather than in erosion score. In the present study, there was no significant group difference in physical function assessed by HAQ, during the 8 years.

The data presented here indicate that routine monitoring not only of radiologic damage but also of swelling and tenderness in the feet is necessary for an adequate evaluation of the disease activity.

A strength of this study is the large number of patients from a well-controlled cohort of early RA patients followed prospectively long-term with a structured protocol including radiographs.

A weakness is that, as this is a post-hoc study, we could not separate the joint counts for swollen and tender joints in the wrists and hands from the 28 joint count included in DAS28.

Summary and conclusions Joint destruction over time was found to be more pronounced in the feet than in the hands, predominantly in younger patients with a negative test for RF and/or antiCCP and who were smokers. Erosions at diagnosis, limited to the feet, were associated with low disease activity by DAS28 at baseline, due to the fact that inflammation limited to the feet is not reflected by DAS28. These observations have relevance for the evaluation of disease activity and joint damage progression and for treatment decisions. Inclusion of the feet in a score like DAS28 would conceivably improve the validity of this established disease activity measure.

This study highlights the importance of evaluating the feet in patients with RA, both with clinical examinations and imaging and lends support to the notion that seropositivity and smoking are risk factors for erosive disease. Further studies of patients with no erosive disease are needed — do these patients have RA or a different disease?

Acknowledgment We thank all the members of the BARFOT study group.

Authors' contributions All authors participated in the design the study, which were analyzed by MA. All authors contributed to interpretation of the data and were involved in drafting the manuscript or revising it critically. All authors approved the final version of the manuscript to be published.
Funding Open access funding provided by Lund University. This study was supported by grants from the Swedish Rheumatism Association and the Foundation for Assistance to Disabled People in Skåne (Stiftelsen för Bistånd åt Rörelsehindrade i Skåne).

Data availability The data analyzed during the current study are available from the corresponding author on reasonable request.

\section{Compliance with ethical standards}

Ethics approval Ethical approval for the present study was obtained (EPN 2014/1986-31/1). Lund University LU 154-95 and 398-01; Göteborg University Gbg M 45-95, Ö 282-01; Linköping University Li 123-95 and 01-263.

Consent to participate and for publication All patients gave their informed consent and the study was performed in accordance with the Declaration of Helsinki.

Disclosures None

Code availability Not applicable

Open Access This article is licensed under a Creative Commons Attribution 4.0 International License, which permits use, sharing, adaptation, distribution and reproduction in any medium or format, as long as you give appropriate credit to the original author(s) and the source, provide a link to the Creative Commons licence, and indicate if changes were made. The images or other third party material in this article are included in the article's Creative Commons licence, unless indicated otherwise in a credit line to the material. If material is not included in the article's Creative Commons licence and your intended use is not permitted by statutory regulation or exceeds the permitted use, you will need to obtain permission directly from the copyright holder. To view a copy of this licence, visit http://creativecommons.org/licenses/by/4.0/.

\section{References}

1. van Gestel AM, Prevoo ML, van't Hof MA, van Rijswijk MH, van de Putte LB, van Riel PL (1996) Development and validation of the European League Against Rheumatism response criteria for rheumatoid arthritis. Comparison with the preliminary American College of Rheumatology and the World Health Organization/ International League Against Rheumatism Criteria. Arthritis Rheum 39(1):34-40. https://doi.org/10.1002/art.1780390105

2. Michelson J, Easley M, Wigley FM, Hellmann D (1994) Foot and ankle problems in rheumatoid arthritis. Foot Ankle Int 15(11):608 613. https://doi.org/10.1177/107110079401501106

3. Erickson AR, Cannella AC, Mikuls TR (eds) (2016) Clinical features of rheumatoid arthritis. Kelley and Firestein's textbook of rheumatology. Elsevier, Philadelphia

4. van der Leeden M, Steultjens M, Dekker JH, Prins AP, Dekker J (2006) Forefoot joint damage, pain and disability in rheumatoid arthritis patients with foot complaints: the role of plantar pressure and gait characteristics. Rheumatology (Oxford) 45(4):465-469. https://doi.org/10.1093/rheumatology/kei186

5. van der Leeden M, Steultjens MP, Ursum J, Dahmen R, Roorda LD, Schaardenburg DV, Dekker J (2008) Prevalence and course of forefoot impairments and walking disability in the first eight years of rheumatoid arthritis. Arthritis Rheum 59(11):1596-1602. https:// doi.org/10.1002/art.24188 
6. Balint GP, Korda J, Hangody L, Balint PV (2003) Regional musculoskeletal conditions: foot and ankle disorders. Best Pract Res Clin Rheumatol 17(1):87-111. https://doi.org/10.1016/s15216942(02)00103-1

7. Otter SJ, Lucas K, Springett K, Moore A, Davies K, Cheek L, Young A, Walker-Bone K (2010) Foot pain in rheumatoid arthritis prevalence, risk factors and management: an epidemiological study. Clin Rheumatol 29(3):255-271. https://doi.org/10.1007/s10067009-1312-y

8. van der Leeden M, Steultjens MP, van Schaardenburg D, Dekker J (2010) Forefoot disease activity in rheumatoid arthritis patients in remission: results of a cohort study. Arthritis Res Ther 12(1):R3. https://doi.org/10.1186/ar2901

9. Chew LC, Chandra Mohan P, Chan LP, Fong KY, Thumboo J (2016) Use of magnetic resonance imaging in detecting subclinical synovitis in rheumatoid arthritis and correlation of imaging findings with interleukin-18 levels. Int J Rheum Dis 19(8):790-798. https:// doi.org/10.1111/1756-185X.12448

10. Forslind K, Svensson B (2016) MRI evidence of persistent joint inflammation and progressive joint damage despite clinical remission during treatment of early rheumatoid arthritis. Scand $\mathrm{J}$ Rheumatol 45(2):99-102. https://doi.org/10.3109/03009742.2015. 1070902

11. Haavardsholm EA, Lie E, Lillegraven S (2012) Should modern imaging be part of remission criteria in rheumatoid arthritis? Best Pract Res Clin Rheumatol 26(6):767-785. https://doi.org/10.1016/ j.berh.2012.10.004

12. Prevoo ML, van't Hof MA, Kuper HH, van Leeuwen MA, van de Putte LB, van Riel PL (1995) Modified disease activity scores that include twenty-eight-joint counts. Development and validation in a prospective longitudinal study of patients with rheumatoid arthritis. Arthritis Rheum 38(1):44-48. https://doi.org/10.1002/art. 1780380107

13. van Gestel AM, Haagsma CJ, van Riel PL (1998) Validation of rheumatoid arthritis improvement criteria that include simplified joint counts. Arthritis Rheum 41(10):1845-1850. https://doi.org/ 10.1002/1529-0131(199810)41:10<1845::AID-ART17>3.0.CO;2$\mathrm{K}$

14. Arnett FC, Edworthy SM, Bloch DA, McShane DJ, Fries JF, Cooper NS, Healey LA, Kaplan SR, Liang MH, Luthra HS et al (1988) The American Rheumatism Association 1987 revised criteria for the classification of rheumatoid arthritis. Arthritis Rheum 31(3):315-324. https://doi.org/10.1002/art.1780310302

15. Andersson ML, Forslind K, Hafstrom I (2015) Comparing five year out-come in two cohorts of patients with early rheumatoid arthritis a BARFOT study. Open Rheumatol J 9:8-15. https://doi.org/10. 2174/1874312901409010008

16. Hafstrom I, Ajeganova S, Andersson ML, Bala SV, Bergman S, Bremander A, Forslind K, Malm K, Svensson B (2019) A Swedish register-based, long-term inception cohort study of patients with rheumatoid arthritis - results of clinical relevance. Open Access Rheumatol 11:207-217. https://doi.org/10.2147/OARRR.S218448

17. Westergren A (1921). Acta med scand (54):247

18. Ekdahl C, Eberhardt K, Andersson SI, Svensson B (1988) Assessing disability in patients with rheumatoid arthritis. Use of a Swedish version of the Stanford Health Assessment Questionnaire. Scand J Rheumatol 17(4):263-271. https://doi.org/10.3109/ 03009748809098795

19. van der Heijde D (2000) How to read radiographs according to the Sharp/van der Heijde method. J Rheumatol 27(1):261-263

20. van der Heijde DM, van Leeuwen MA, van Riel PL, van de Putte LB (1995) Radiographic progression on radiographs of hands and feet during the first 3 years of rheumatoid arthritis measured according to Sharp's method (van der Heijde modification). J Rheumatol 22(9): 1792-1796
21. Hulsmans HM, Jacobs JW, van der Heijde DM, van AlbadaKuipers GA, Schenk Y, Bijlsma JW (2000) The course of radiologic damage during the first six years of rheumatoid arthritis. Arthritis Rheum 43(9):1927-1940. https://doi.org/10.1002/15290131(200009)43:9<1927::AID-ANR3>3.0.CO;2-B

22. Plant MJ, Jones PW, Saklatvala J, Ollier WE, Dawes PT (1998) Patterns of radiological progression in early rheumatoid arthritis: results of an 8 year prospective study. J Rheumatol 25(3):417-426

23. Krol A, Garred P, Heegaard NH, Christensen AF, Hetland ML, Stengaard-Pedersen K, Junker P, Madsen HO, Lottenburger T, Ellingsen T, Andersen LS, Hansen I, Pedersen JK, Svendsen AJ, Tarp U, Podenphant J, Lindegaard H, Ostergaard M, HorslevPetersen K, Jacobsen S (2015) Interactions between smoking, increased serum levels of anti-CCP antibodies, rheumatoid factors, and erosive joint disease in patients with early, untreated rheumatoid arthritis. Scand J Rheumatol 44(1):8-12. https://doi.org/10. 3109/03009742.2014.918651

24. Bakker MF, Jacobs JW, Kruize AA, van der Veen MJ, van BoomaFrankfort C, Vreugdenhil SA, Bijlsma JW, Lafeber FP, Welsing PM (2012) Misclassification of disease activity when assessing individual patients with early rheumatoid arthritis using disease activity indices that do not include joints of feet. Ann Rheum Dis 71(6): 830-835. https://doi.org/10.1136/annrheumdis-2011-146670

25. Wechalekar MD, Lester S, Hill CL, Lee A, Rischmueller M, Smith MD, Walker JG, Proudman SM (2016) Active foot synovitis in patients with rheumatoid arthritis: unstable remission status, radiographic progression, and worse functional outcomes in patients with foot synovitis in apparent remission. Arthritis Care Res 68(11):1616-1623. https://doi.org/10.1002/acr.22887

26. Liao KP, Weinblatt ME, Cui J, Iannaccone C, Chibnik LB, Lu B, Coblyn JS, Shadick NA, Solomon DH (2011) Clinical predictors of erosion-free status in rheumatoid arthritis: a prospective cohort study. Rheumatology (Oxford) 50(8):1473-1479. https://doi.org/ 10.1093/rheumatology/ker129

27. Fex E, Jonsson K, Johnson U, Eberhardt K (1996) Development of radiographic damage during the first 5-6 yr of rheumatoid arthritis. A prospective follow-up study of a Swedish cohort. Br J Rheumatol 35(11):1106-1115. https://doi.org/10.1093/rheumatology/35.11. 1106

28. Amaya-Amaya J, Calixto OJ, Saade-Lemus S, Calvo-Paramo E, Mantilla RD, Rojas-Villarraga A, Anaya JM (2015) Does nonerosive rheumatoid arthritis exist? A cross-sectional analysis and a systematic literature review. Semin Arthritis Rheum 44(5):489 498. https://doi.org/10.1016/j.semarthrit.2014.09.006

29. Forslind K, Svensson B (2019) Repair of bone erosions in rheumatoid arthritis: a systematic literature review. Scand J Rheumatol 48(6):448-453. https://doi.org/10.1080/03009742.2019.1606274

30. Wan SW, He HG, Mak A, Lahiri M, Luo N, Cheung PP, Wang W (2016) Health-related quality of life and its predictors among patients with rheumatoid arthritis. Appl Nurs Res 30:176-183. https:// doi.org/10.1016/j.apnr.2015.07.004

31. Luukkainen R, Sokka T, Kautiainen H, Hannonen P, Laasonen L, Leirisalo-Repo M, Korpela M, Julkunen H, Puolakka K, Blafield H, Kauppi M, Mottonen T, Group FI-RT (2007) Prognosis of 5-year radiographic erosions of the wrist according to early, late, and persistent wrist swelling or tenderness in patients with early rheumatoid arthritis. J Rheumatol 34(1):50-53

32. Sokka T, Kautiainen H, Mottonen T, Hannonen P (2003) Erosions develop rarely in joints without clinically detectable inflammation in patients with early rheumatoid arthritis. J Rheumatol 30(12): 2580-2584

33. Smolen JS, Breedveld FC, Burmester GR, Bykerk V, Dougados M, Emery P, Kvien TK, Navarro-Compan MV, Oliver S, Schoels M, Scholte-Voshaar M, Stamm T, Stoffer M, Takeuchi T, Aletaha D, Andreu JL, Aringer M, Bergman M, Betteridge N, Bijlsma H, Burkhardt H, Cardiel M, Combe B, Durez P, Fonseca JE, 
Gibofsky A, Gomez-Reino JJ, Graninger W, Hannonen P, Haraoui B, Kouloumas M, Landewe R, Martin-Mola E, Nash P, Ostergaard M, Ostor A, Richards P, Sokka-Isler T, Thorne C, Tzioufas AG, van Vollenhoven R, de Wit M, van der Heijde D (2016) Treating rheumatoid arthritis to target: 2014 update of the recommendations of an international task force. Ann Rheum Dis 75(1):3-15. https://doi. org/10.1136/annrheumdis-2015-207524

34. McInnes IB, O'Dell JR (2010) State-of-the-art: rheumatoid arthritis. Ann Rheum Dis 69(11):1898-1906. https://doi.org/10.1136/ard. 2010.134684

35. Combe B, Cantagrel A, Goupille P, Bozonnat MC, Sibilia J, Eliaou JF, Meyer O, Sany J, Dubois A, Daures JP, Dougados M (2003) Predictive factors of 5-year health assessment questionnaire disability in early rheumatoid arthritis. J Rheumatol 30(11):2344-2349

36. Odegard S, Landewe R, van der Heijde D, Kvien TK, Mowinckel P, Uhlig T (2006) Association of early radiographic damage with impaired physical function in rheumatoid arthritis: a ten-year, longitudinal observational study in 238 patients. Arthritis Rheum 54(1):68-75. https://doi.org/10.1002/art.21548

37. Andersson MLE, Forslind K, Hafstrom I, Group BS (2017) Patients with early rheumatoid arthritis in the 2000s have equal disability and pain despite less disease activity compared with the 1990s: data from the BARFOT study over 8 years. J Rheumatol 44(6):723-731. https://doi.org/10.3899/jrheum.161235

38. Maillefert JF, Combe B, Goupille P, Cantagrel A, Dougados M (2004) The 5-yr HAQ-disability is related to the first year's changes in the narrowing, rather than erosion score in patients with recentonset rheumatoid arthritis. Rheumatology (Oxford) 43(1):79-84. https://doi.org/10.1093/rheumatology/keg465

Publisher's note Springer Nature remains neutral with regard to jurisdictional claims in published maps and institutional affiliations. 